\title{
Patientenanruf
}

\section{Wie sich die telefonische Erinnerung bezahlt macht}

\author{
Viele Ärzte nutzen das Recall-System nicht konsequent: Dabei kann \\ sich der Kontakt zum Patienten, um ihn an einen Termin oder eine \\ Vorsorgeuntersuchung zu erinnern, auch für den Arzt lohnen.
}

nnerhalb der vielen Möglichkeiten der Kundenbindung in der Arztpraxis nimmt das Recall-Verfahren, also das Wiederbestellsystem, eine zentrale Stellung ein. Mit einem solchen Verfahren lässt sich aktive Patientenbindung betreiben und eine systematische Umsatzsteigerung beziehungsweise Umsatzsicherung erwirken.

Die verschiedenen EDV-Systeme für Arztpraxen besitzen eine entsprechende Funktion für Recall-Verfahren. Recall kann dabei in doppeltem Sinn verstanden werden:

_als Erinnerung an bereits vereinbarte Termine, vor allem bei langfristig geplanten Terminen,

_als Erinnerung an anstehende Untersuchungen oder Vorsorge mit der anschließenden konkreten Vereinbarung eines Termins.

\section{Gute Organisation wichtig}

Zuerst sollten Sie sich über die Funktionsweise des Recall-Verfahrens in Ihrer EDV informieren. Daraus ergeben sich die organisatorischen Maßnahmen zur Implementierung des Verfahrens. Dann ist zu klären, wie die entsprechenden Termine einzugeben sind und wie das Praxisteam auf die fälligen Termine aufmerksam gemacht wird. Sie können sich zum Beispiel jeden Montag eine Liste der fälligen Termine mit Patienten auf dem Bildschirm anzeigen lassen. Idealerweise haben Sie die Einstellungen so vorgenommen, dass die Telefonnummer des betreffenden Patienten gleich mit angegeben wird. Dann muss ein Teammitglied nur noch die Patienten anrufen oder anmailen und über den fälligen Termin informieren oder einen Praxistermin vergeben.

Im nächsten Schritt sollten Sie definieren, für welche Bereiche Sie ein RecallVerfahren nutzen wollen. Sinnvoll ist es zum Beispiel bei Kontrolluntersuchungen sowie Krebsvor- oder -nachsorge.

Dann müssen Sie auch klären, wie gegebenenfalls Folgetermine schnell sichtbar gemacht werden können, also bei Aufruf eines Patienten sofort auf dem Bildschirm erscheinen. Das ist wichtig, damit Sie im Praxisalltag sofort erkennen können, ob der betreffende Patient überhaupt am Recall-Verfahren teilnimmt. Wenn das Recall-Verfahren implementiert ist, genügt ein Blick, und jedes Teammitglied erkennt sofort, dass ein Folgetermin eingetragen, also der betreffende Patient schon aktiv im System ist. Findet sich in dem betreffenden Fenster kein Eintrag, dann muss oder kann der Patient aufgenommen werden. Zumindest erkennen Sie sofort, dass bei dem betreffenden Patienten wohl noch keine der Untersuchungen oder Leistungen aus Ihrer praxisindividuellen Liste erbracht worden ist. Das zeigt Ihnen dann Handlungsbedarf an. Dazu ist es notwendig, dass für alle Patienten, bei denen zum Beispiel eine Vorsorgeuntersuchung in Ihrer Praxis durchgeführt wurde, auch ein entsprechender Folgetermin (in einem Jahr) eingetragen wird.

Um diejenigen Patienten anzusprechen, die möglicherweise überhaupt noch nicht zu einer Präventionsleistung wie der Krebsvorsorge in Ihrer Praxis waren, sollten Sie über die Suchfunktion
„Alter“, in Ihrer EDV alle diejenigen Patienten herausfiltern, die Anspruch auf eine solche Vorsorgeuntersuchung haben und diese dann darüber informieren. Mit einem Recall-Verfahren lassen sich auf diese Art und Weise zum Teil enorme Umsätze aus dem Bereich der nicht budgetierten Gesamtvergütung sichern.

\section{Unbudgetiertes Honorar}

Wenn Sie nach einem funktionierenden Recall-Verfahren arbeiten, können Sie einfach den Wiederholungstermin oder Erinnerungstermin aktuell vermerken. Das ist auch deshalb sehr wichtig, da mit jeder Vorsorgeuntersuchung im GKVSystem unbudgetiertes Honorar erwirtschaftet wird.

Bei optimaler Organisation wird Ihr Recall-Verfahren immer ausgefeilter und vollständiger. Parallel dazu erkennen Sie, dass sich aus diesem System immer mehr Wiederholungstermine mit Ihren Patienten organisieren lassen. Die Folge ist dann eine erhöhte Frequenz der Inanspruchnahme. Das ist Kundenorientierung und Kundenbindung zum beiderseitigen Nutzen.

\section{Einverständniserklärung nötig}

Oft genannte Probleme im Zusammenhang mit dem Recall-System sind die nicht vorhandenen Telefonnummern von Patienten oder auch die notwendige Einverständniserklärung Ihrer Patienten. Diese benötigen Sie, wenn Sie diese über fällige Termine benachrichtigen wollen.

Für sinnvolle Vorsorge- oder Kontrolluntersuchungen geben Patienten aber in der Regel gerne ihr Einverständnis. Und wenn dann noch der Kontaktweg gewählt wird, der dem Patienten am meisten liegt - Telefon, SMS oder E-Mail -, erhöht das zusätzlich die Akzeptanz des Recalls.

Dr. Dr. Peter Schlüter 